\title{
Electron Screening in Nuclear Astrophysics
}

\author{
Matej Lipoglavsek and Aleksandra Cvetinović \\ Jozef Stefan Institute, SI-1000 Ljubljana, Slovenia
}

\begin{abstract}
Electron screening in an important effect that cannot be neglected in nuclear astrophysics, since it influences nuclear reaction cross sections at low energies. We are trying to understand why most measurements in inverse kinematics on solid targets give electron screening potentials more than an order of magnitude above predictions. Below we report our latest results on electron screening in nuclear reactions ${ }^{1} \mathrm{H}\left({ }^{14} \mathrm{~N}, \gamma\right){ }^{15} \mathrm{O}$ and ${ }^{2} \mathrm{H}\left({ }^{19} \mathrm{~F}, \mathrm{p}\right)^{20} \mathrm{~F}$ in both inverse and normal kinematics. The analysis is in progress.
\end{abstract}

\section{Introduction}

Electron screening is an effect that enhances nuclear reaction probabilities at low energies, when both reactants are positively charged nuclei. It was predicted in the very early days of the field of nuclear astrophysics [1]. Later, the experimentalists confirmed the existence of the effect in the laboratory. However, the magnitude of the effect appeared to be much larger than the theory predicted [2]. This discrepancy still exists almost 20 years later, which leads many nuclear astrophysicists to disbelieve the measurements. Despite the fact that some experimental mistakes have been made in the past, especially in the interpretation of the measured results [3,4], several different groups are now reporting similar results [5-10]. Therefore, the problem remains and needs to be solved, before one can apply electron screening to stellar plasmas, which is completely unknown. The extremely simplified theoretical approach assumes that the atomic electrons form a uniformly charged spherical shell with a radius $R_{a}$, which is the atomic radius [2]. The reaction cross section $\sigma$ is then enhanced with the enhancement factor $f$ defined as the ratio of the screened and bare cross sections as

$$
f(E)=\frac{\sigma\left(E+U_{e}\right)}{\sigma(E)},
$$

where E represents the energy in the center of mass system and $U_{e}$ the electron screening potential [2]. From the known position of the electrons in the atom, $U_{e}$ can be simply calculated as

$$
U_{e}=\frac{Z_{1} Z_{2} e^{2}}{4 \pi \varepsilon_{0} R_{a}}
$$


where $Z_{1}$ and $Z_{2}$ are the charge numbers of the projectile and target nuclei [2]. This result is called the adiabatic limit and represents the maximum value for $U_{e}$. While the theory predicts an electron screening potential independent of the target host material, measurements report a strong dependence of cross section enhancement on the target host, which further substantiates the above-mentioned disbelief in measurements. When gaseous targets are used, the electron screening potential usually remains within the adiabatic limit. However, when target nuclei are implanted into a solid lattice, the $U_{e}$ is often more than an order of magnitude above predictions. Moreover, the magnitude of screening seems to depend on the metallurgy of the solid lattice. In addition, the radiation damage to the solid target during the measurement further enhances electron screening. To ease the discussion below we define here also the astrophysical $S$-factor as

$$
\sigma(E)=\frac{S(E)}{E} e^{-2 \pi \eta},
$$

where $\eta$ is the Sommerfeld parameter [2]. Our group at Jozef Stefan Institute is now trying to understand electron screening in solid targets, before we could apply the effect to stellar plasmas. We are currently focusing on reactions on hydrogen isotopes implanted in different titanium lattices, since a peculiar dependence of electron screening on deuterium concentration in titanium has previously been reported [11]. We will try to detect a difference in electron screening between two different titanium targets and then try to find, in which parameters those targets differ. We will apply thermal desorption spectroscopy, NMR spectroscopy, Raman spectroscopy, X-ray diffraction and possibly other techniques to determine the differences in the position of hydrogen isotopes in the titanium lattice and the condition of the lattice itself. The nuclear reactions that we are using for the studies of electron screening are ${ }^{1} \mathrm{H}\left({ }^{14} \mathrm{~N}, \gamma\right){ }^{15} \mathrm{O}$ and ${ }^{2} \mathrm{H}\left({ }^{19} \mathrm{~F}, \mathrm{p}\right){ }^{20} \mathrm{~F}$.

\section{Experiments}

At Jozef Stefan Institute we measured the cross section for the reaction between deuterium and ${ }^{19} \mathrm{~F}$ nuclei in inverse kinematics. The experiment was performed at the $2 \mathrm{MV}$ Tandetron accelerator delivering intense ${ }^{19} \mathrm{~F}$ beams with energies between 3090 and 9200 $\mathrm{keV}$. The deuterium targets were prepared by ion implantation with Tectra IonEtch ion gun at $3.5 \mathrm{kV}$. The deuterium was implanted into thick titanium sheets. The deuterium distribution in titanium was very non-uniform after implantation. Therefore, we performed quantitative depth profiling of deuterium with the Nuclear Reaction Analysis technique for each implanted target. We used the reaction ${ }^{2} \mathrm{H}\left({ }^{3} \mathrm{He}, \mathrm{p}\right){ }^{4} \mathrm{He}$ and measured the high energy protons emitted in the reaction. One of the measured deuterium depth profiles is shown in Fig. 1. The profile shows high deuterium concentrations at depths much higher than the implantation depth at $3.5 \mathrm{keV}$. This shows that deuterium diffuses into titanium and is trapped by the defects in the titanium crystal lattice caused by previous irradiation with fluorine beams of different energies. For more on the depth profiling see e.g. ref. [12].

To gain more insight into deuterium distribution in titanium, we performed thermal desorption spectroscopy (TDS) of hydrogen isotopes. With this technique the deuterium implanted titanium sheet is heated at a constant rate. The gases released from titanium are then measured and analyzed for their mass. Fig. 2 shows the number of ions with mass 4 atomic mass units released from titanium at different sample temperatures for two different deuterium implanted titanium samples. The figure shows that deuterium is trapped at least at three different trapping sites, having three different binding energies. The only difference between the two samples was different previous radiation damage. 


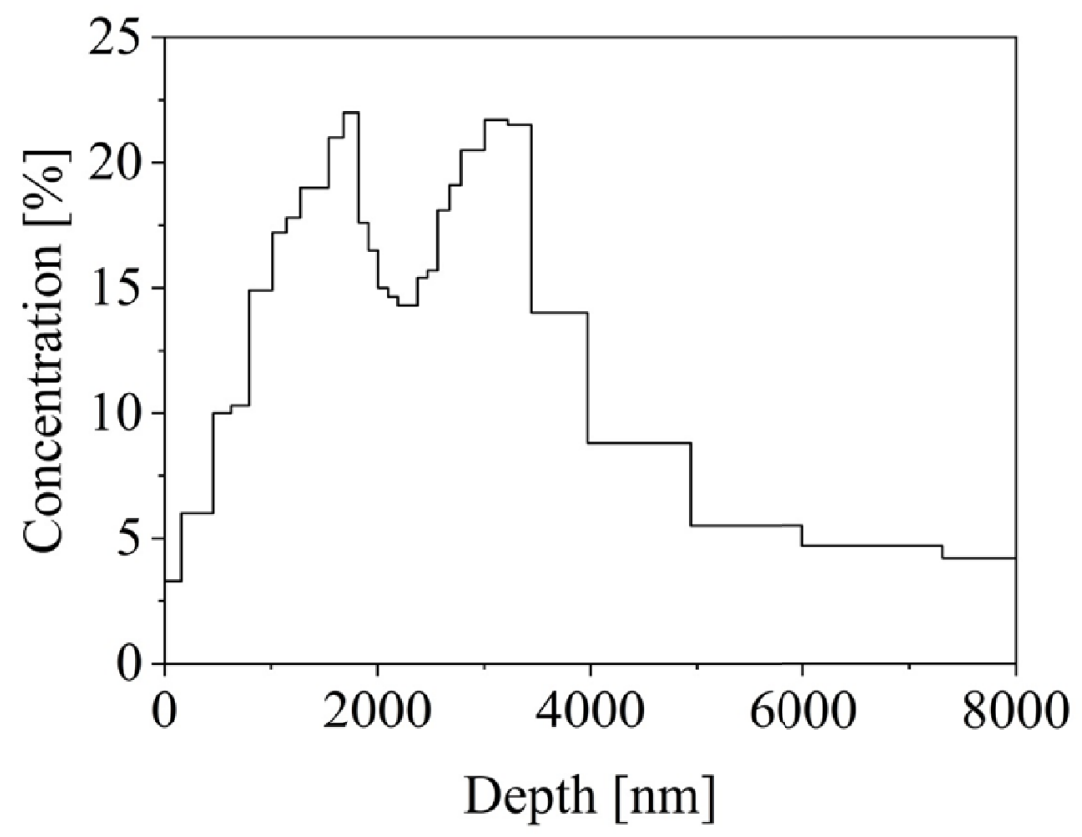

Fig. 1. Deuterium concentration relative to titanium as a function of the depth in titanium. The two peaks show the maxima of previous radiation damage. The range of $3.5 \mathrm{keV}$ deuterium ions in titanium is $41 \mathrm{~nm}[13]$.

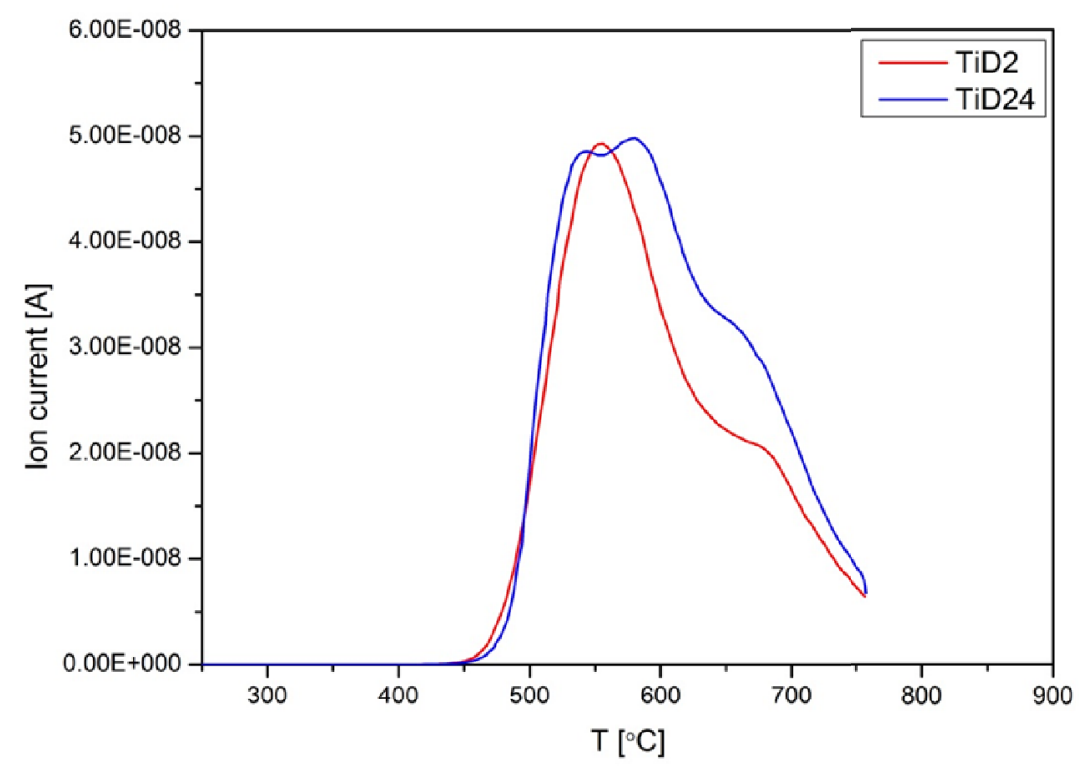

Fig. 2. Mass 4 a.m.u. ion current as a function of titanium sample temperature for two different titanium samples marked as TiD2 and TiD24. The only difference between the two samples is different previous radiation damage. 
In the nuclear reaction ${ }^{2} \mathrm{H}\left({ }^{19} \mathrm{~F}, \mathrm{p}\right){ }^{20} \mathrm{~F}$ we produced the radioactive isotope ${ }^{20} \mathrm{~F}$, which decays with a half-life of $11 \mathrm{~s}$ [14]. In the decay, a $\gamma$-ray with an energy of $1634 \mathrm{keV}$ is emitted with a branching ratio of $99.1 \%$ [14]. By measuring the count rate of this $\gamma$-ray we could determine the cross section for the above reaction. A germanium detector was placed at the angle of $135^{\circ}$ with respect to the beam direction. The target-detector distance was 5.7 $\mathrm{cm}$. The intrinsic detector efficiency was $53 \%$ relative to a 3" by 3" NaI detector. The beam current was measured on an electrically insulated target chamber. The preliminary results are shown in Fig. 3, which shows the cross section enhancement factor $f$. The enhancement factor was calculated by assuming a constant $S$-factor, since the reaction cross section is not known at these energies. This is certainly not correct, and the bare $S$-factor will have to be determined. We will do this by measuring the same reaction in normal kinematics, where we do not expect to observe large electron screening. We would have done that already, unless it was nearly impossible to obtain a low energy deuterium beam anywhere in Europe these days. This is due to the concerns of radiation protection and some political issues about the interference of nuclear and atomic physics, completely contrary to our understanding of open science. Our current plan is to try to measure the cross section in normal kinematics at Max Planck Institute in Garching. The result of our assumption about the $S$-factor is probably also the extremely high electron screening potential $(220 \mathrm{keV})$ that shows up in the calculation. For comparison, the adiabatic limit for the same reaction is 2.2 $\mathrm{keV}$.

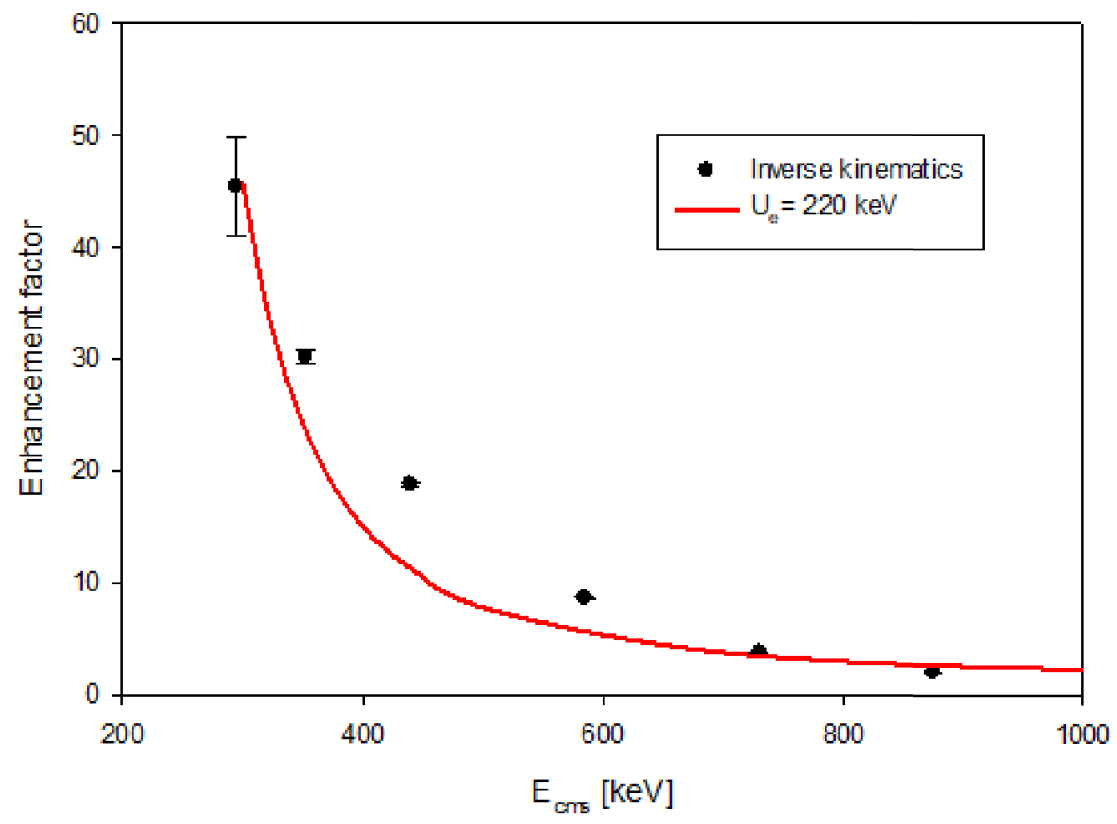

Fig. 3. Cross section enhancement as a function of center of mass energy for the reaction ${ }^{2} \mathrm{H}\left({ }^{19} \mathrm{~F}, \mathrm{p}\right)^{20} \mathrm{~F}$. The black circles are our measurements on a deuterium implanted titanium target. The red line represents a calculation with $U_{e}=220 \mathrm{keV}$ and assumes a constant $S$-factor.

The reaction ${ }^{1} \mathrm{H}\left({ }^{14} \mathrm{~N}, \gamma\right){ }^{15} \mathrm{O}$ was recently studied at Helmholz Zentrum Dresden Rossendorf. Since this reaction is with protons we were able to measure it in both normal and inverse kinematics. We measured the resonance strength of the resonance at $259 \mathrm{keV}$ center of mass energy [15]. For calibration and to deduce the hydrogen content in the target 
we used the resonances of the reaction ${ }^{1} \mathrm{H}\left({ }^{15} \mathrm{~N}, \alpha \gamma\right){ }^{12} \mathrm{C}$ at 403 and $841 \mathrm{keV}$ center of mass energy and the reaction ${ }^{27} \mathrm{Al}(\mathrm{p}, \gamma){ }^{28} \mathrm{Si}$ at $992 \mathrm{keV}$ proton energy. The intense proton, ${ }^{14} \mathrm{~N}$ and ${ }^{15} \mathrm{~N}$ beams were delivered by the $3 \mathrm{MV}$ Tandem accelerator at HZDR. The beam intensity was measured on the insulated target with secondary electron suppression. It depended on the energy but was generally between $50 \mathrm{nA}$ for the highest energy with ${ }^{15} \mathrm{~N}$ and $4 \mu \mathrm{A}$ for the lowest one with ${ }^{14} \mathrm{~N}$. The targets were prepared at TU Dresden and at Jozef Stefan Institute by hydrogenation, implantation and evaporation. They were cooled by water in direct contact and were tilted by $55^{\circ}$ with respect to the beam direction. We used five different types of targets, hydrogenated titanium on tantalum backing, implanted thick titanium sheet, implanted graphite sheet, evaporated TiN on tantalum backing, as well as evaporated aluminum on tantalum backing. The $\gamma$ rays were measured by a germanium detector placed about $3 \mathrm{~cm}$ from the target at the angle of $55^{\circ}$ with respect to the beam and by a $\mathrm{LaBr}_{3}$ detector at $90^{\circ}$. The germanium detector intrinsic efficiency was $100 \%$ relative to a 3" by 3" NaI detector. The analysis of the data is in progress. The online result is shown in figure 4 , which shows the $\gamma$-ray yield of the $6175 \mathrm{keV} \gamma$-ray emitted in the decay of the $7556 \mathrm{keV}$ state in ${ }^{15} \mathrm{O}$. This state was populated in the reaction ${ }^{1} \mathrm{H}\left({ }^{14} \mathrm{~N}, \gamma\right){ }^{15} \mathrm{O}$ on a hydrated titanium target.

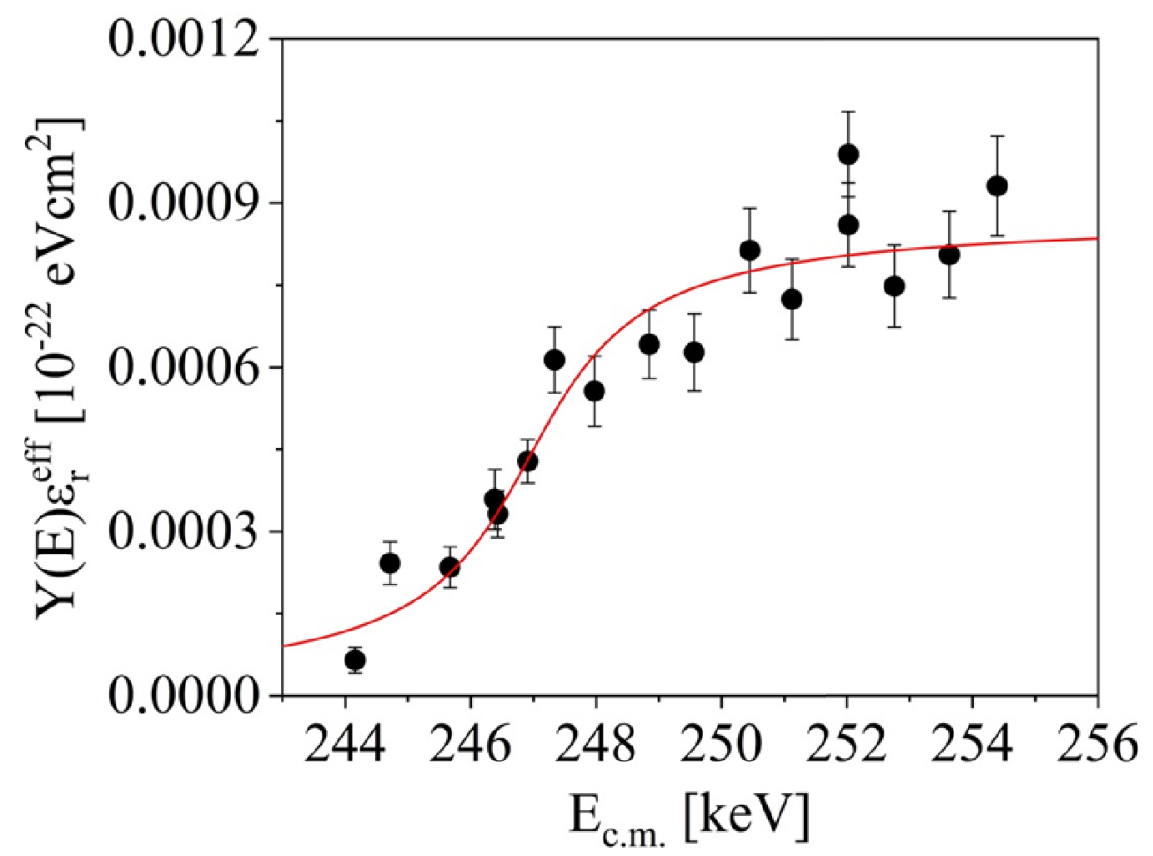

Fig. 4. Gamma-ray yield of the $6175 \mathrm{keV} \gamma$-ray emitted in the decay of the $7556 \mathrm{keV}$ state in ${ }^{15} \mathrm{O}$ as a function of the center of mass energy. The red line represents a fit with the arctan function [10].

\section{Conclusions}

Electron screening in nuclear reactions is an important effect that needs to be taken into account in nuclear astrophysics. Due to the discrepancy between experiments and theory, it is not yet clear how electron screening should be treated in stellar plasmas. In laboratory experiments we observed a large effect in inverse kinematics reactions on hydrogen 
isotopes. For this case and until electron screening is better understood, we suggest to use the following equation to calculate the electron screening potential

$$
U_{e}=Z^{2} \cdot U_{0}
$$

where $U_{0}=0.8 \mathrm{keV}$ is the maximum electron screening potential measured for the ${ }^{2} \mathrm{H}(\mathrm{d}, \mathrm{p})^{3} \mathrm{H}$ reaction [5]. Perhaps a similar equation should also be used to treat electron screening in the Universe. In view of the above, we will try to calculate the influence of electron screening in primordial nucleosynthesis.

\section{References}

1. E. E. Salpeter, Austr. J. Phys. 7 (1954) 373.

2. H. J. Assenbaum, K. Langanke and C. Rolfs, Z. Phys. A 327 (1987) 461.

3. K. U. Kettner et al., J. Phys. G 32 (2006) 489.

4. J. Gajevic et al., Eur. Phys. J. A49 (2013) 70.

5. F. Raiola et al., Eur. Phys. J. A19 (2004) 283.

6. J. Kasagi, Prog. Theo. Phys. Suppl. 154 (2004) 365.

7. A. Huke et al., Phys. Rev. C 78 (2008) 015803.

8. J. Cruz et al., Phys. Lett. B 624 (2005) 181; J Cruz et al., J. Phys. G 35 (2008) 014004.

9. M. Lipoglavsek et al., Eur. Phys. J. A44 (2010) 71.

10. A. Cvetinovic et al., Phys.Rev. C 92, (2015) 065801.

11. F. Raiola et al., J. Phys. G 31 (2005) 1141.

12. I. Tisma et al., Eur.Phys.J. A55, (2019) 137.

13. J.F. Ziegler, J.P. Biersack, M.D. Zeigler, The Stopping and range of Ions in matter, Lulu press Co., Morrisville, NC, USA, 2008; http://www.srim.org.

14. D. R. Tilley et al., Nucl. Phys. A636 (1998) 249.

15. M. Marta et al., Phys. Rev. C 81 (2010) 055807. 